\title{
Nondestructive 3D Nanoscale X-ray Imaging of Solid Oxide Fuel Cells in the Laboratory
}

\author{
Stephen T. Kelly ${ }^{1 *}$, Sandrine Ricote ${ }^{2}$, Peter Weddle ${ }^{2}$, Alexis Dubois ${ }^{2}$, Benjamin Kee ${ }^{2}$, William Harris ${ }^{1}$, \\ John Berger ${ }^{2}$ and Robert J. Kee ${ }^{2}$ \\ 1. Carl Zeiss X-ray Microscopy, Inc., Pleasanton, CA, USA. \\ 2. Department of Mechanical Engineering, Colorado School of Mines, Golden, CO, USA. \\ * Corresponding author: steve.kelly@zeiss.com
}

Fuel cells and similar energy devices rely heavily on complex 3D microstructures for efficient operation. However, capturing these intricate features at appropriate length scales and with accessible imaging techniques has remained a challenge. Imaging techniques not only provide direct insight into the complex structures and transport networks that exist, but also provide geometrical input to a host of performance models that rely on experimental observations to tie their results to real-world outcomes. In addition, ideal experimental observations in this framework can track the evolution of sample parameters (microstructure, chemistry, loads, etc) non-destructively over several stages of processing or operation and across multiple length scales.

As an example of this approach, we present here results from non-destructively imaging and analyzing the microstructures of two different fuel cell architectures using x-ray nanotomography. All samples were imaged using a ZEISS Xradia 810 Ultra x-ray microscope after being prepared using a combination of laser ablation and focused ion beam milling to create samples of the correct size and shape (cylinders $\sim 20 \mu \mathrm{m}$ in diameter) for the tomography measurements.

First, we examined a $\mathrm{H}^{+}$-solid oxide fuel cell (or protonic ceramic fuel cell) anode made of $\mathrm{BaCe}_{0.2} \mathrm{Zr}_{0.7} \mathrm{Y}_{0.1} \mathrm{O}_{3-\mathrm{d}}(\mathrm{BCZY}) / \mathrm{Ni}$. The sample was prepared by solid-state reactive sintering as described in [1]. The material contrast and spatial resolution (down to $50 \mathrm{~nm}$ ) of the technique allows for discrimination of discrete Ni and BCZY phases, and identification of gas-phase features such as pores and cracks. Quantitative analyses include measuring and visualizing the triple phase boundary, plotting the trajectory of cracks in 3D, and can be used to build a 3D structural model of the sample for further computational input such as transport or mechanical simulations. Results showing a variety of material properties computed from the imaging results will be presented.

Second, we present an analysis on a $\mathrm{Ni}+\mathrm{ZrO}_{2}: 8 \% \mathrm{Y}_{2} \mathrm{O}_{3}(8 \mathrm{YSZ}) / 8 \mathrm{YSZ} / \mathrm{La}_{1-\mathrm{x}} \mathrm{Sr}_{\mathrm{x}} \mathrm{MnO}_{3}$ (LSM) solid oxide fuel cell stack exposed to air to promote nickel oxidation. The detailed 3D tomographic reconstructions allow for several important material parameters to be quantified. We analyzed the pore and LSM size distribution in the cathode material, examined the connectivity of pores in the electrolyte layer, and were able to identify the different phases and plot the triple phase boundary in the anode.

The 3-dimensional nature of the results presented here reflects the complexity of the 3D electrodes, and enables more advanced analyses inaccessible through 2D imaging alone. Furthermore, the nondestructive nature of the technique opens the door to a wide array of experimental possibilities such as additional imaging or analysis with complementary techniques (such as SEM or FIB-SEM), or imaging the sample after a processing treatment. In sum, these techniques provide valuable data for validation and refinement of the 3D structural models used to predict the performance and failure mechanisms in 
these devices, and help close the loop in this "materials by design" approach.

References:

[1] SM Babiniec, S Ricote and NP Sullivan, Int. J. Hydrogen Energy 40 (2015), p. 9278.

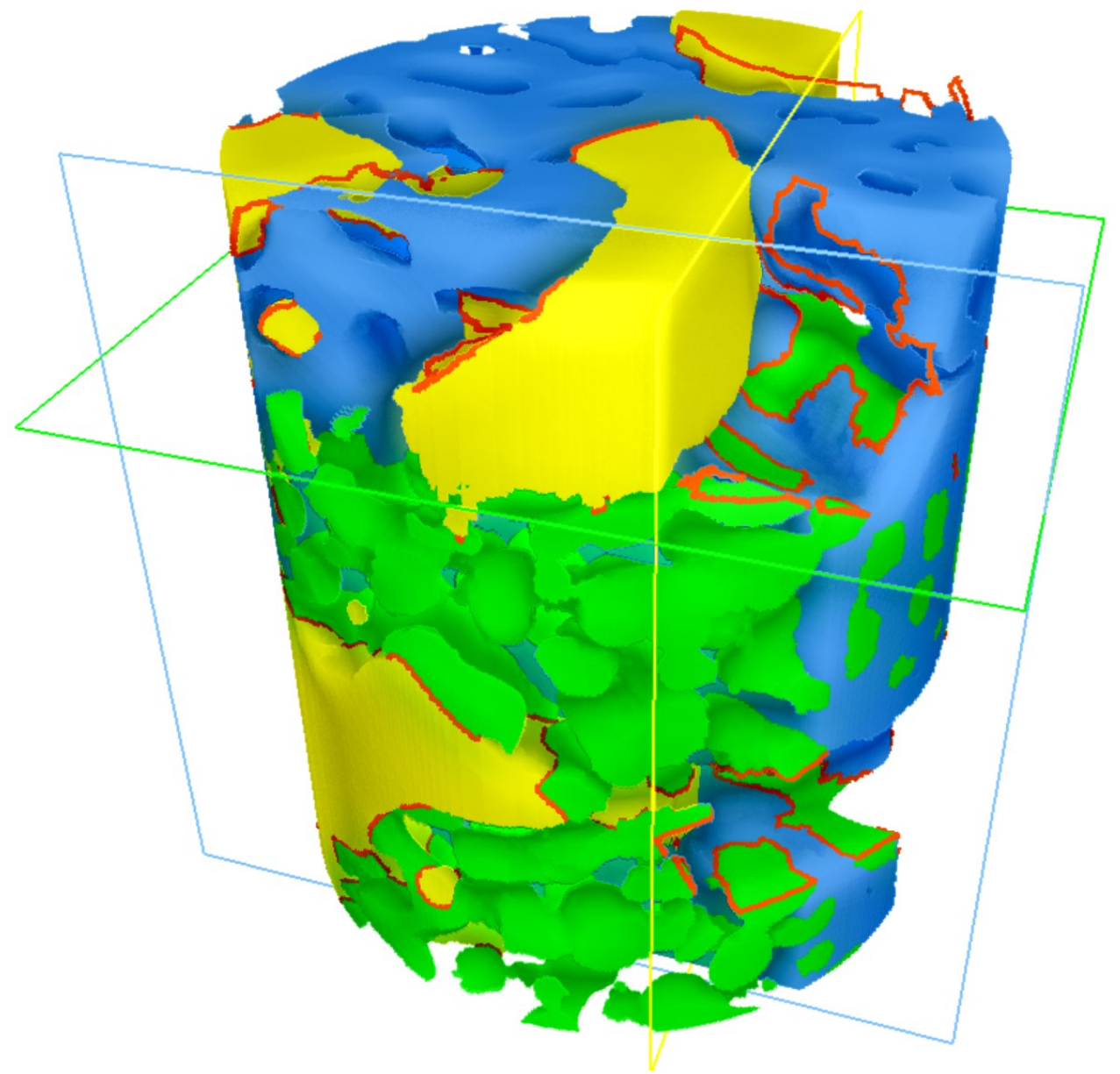

Figure 1. Segmented image of the $\mathrm{H}+$ solid oxide fuel cell anode imaged in this study. Green indicates pore space, blue indicates nickel, yellow indicates the BCZY ceramic phase, and orange indicates the triple phase boundary. The diameter of the analyzed cylinder shown is $16 \mu \mathrm{m}$. 